\title{
Infective Aortitis
}

Key words: infective aortitis, computed tomography, air density spot

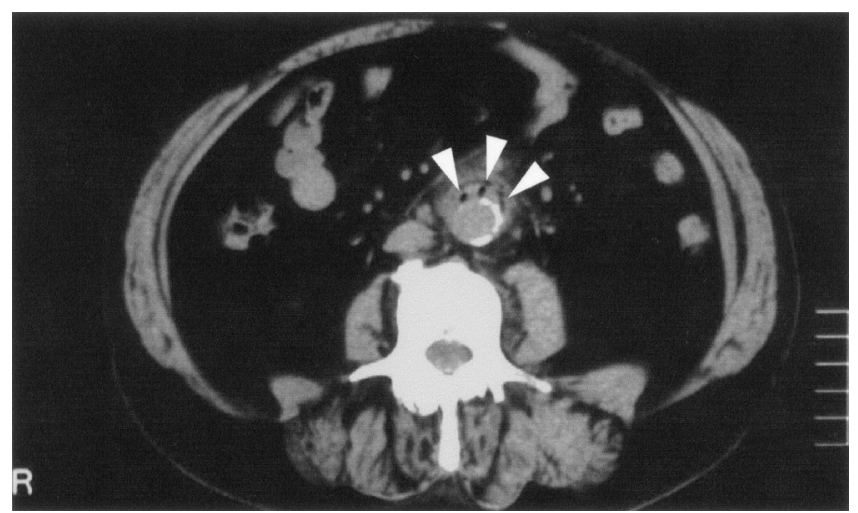

A

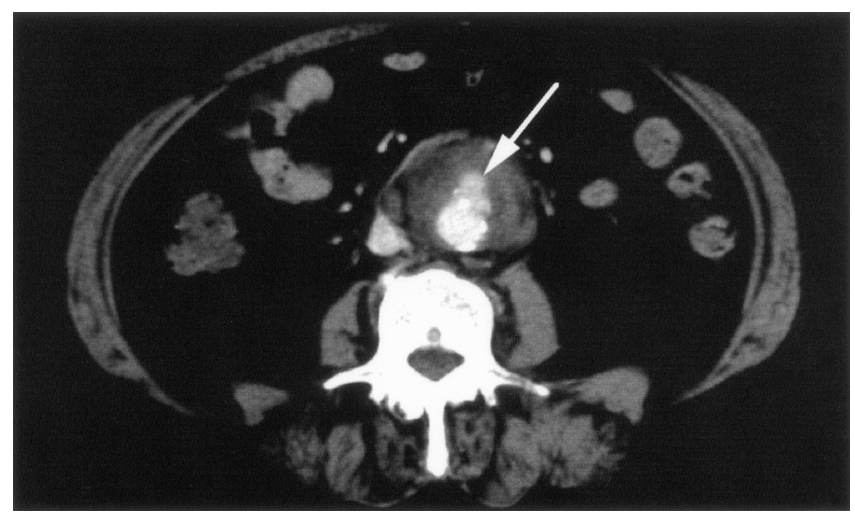

$\mathrm{B}$

Figure 1. Abdominal computed tomography (A, on admission, plain; B, on the 13th hospital day, enhanced). Arrowheads indicate air density spots in the periaortic abscess, and the arrow indicates extravasation of contrast medium into the periaortic abscess.

Infective aortitis, though uncommon, must be recognized as an etiology of fever of patients with advanced atherosclerosis, and early treatment can improve its survival.

A 68-year-old woman was admitted to our hospital because of fever and abdominal pain. She had had a history of rheumatoid arthritis treated with steroid for ten years. On admission, the white blood cell count was $18,800 / \mu 1$ and C-reactive protein level was $25.8 \mathrm{mg} / \mathrm{dl}$. Several blood cultures for bacteria were negative. An abdominal CT revealed a periaortic abscess with air density spots (Fig. 1A). She was diagnosed with infective aortitis and treated with antibiotics. On the 13th hospital day, an enhanced CT demonstrated an increase in periaortic abscess with extravasation of contrast medium (Fig. 1B). Impending rupture of infected aneurysm was clinically diagnosed, and she underwent emergency operation at another hospital. Air density spots in periaortic abscess are useful findings for early diagnosis of infective aortitis.

\section{Tsutomu AraKi and Akikatsu NAKASHIMA}

From the Department of Internal Medicine, Saiseikai Kanazawa Hospital, Kanazawa

Received for publication May 30, 2005; Accepted for publication June 12, 2005

Reprint requests should be addressed to Dr. Tsutomu Araki, the Department of Internal Medicine, Saiseikai Kanazawa Hospital,

Ni 13-6, Akatsuchi-machi, Kanazawa, Ishikawa 920-0353 